Article

\title{
The Effect of Training-Induced Visual Imageability on Electrophysiological Correlates of Novel Word Processing
}

\author{
Laura Bechtold *, Marta Ghio and Christian Bellebaum \\ Institute for Experimental Psychology, Heinrich Heine University Düsseldorf, 40225 Düsseldorf, Germany; \\ Marta.Ghio@hhu.de (M.G.); Christian.Bellebaum@hhu.de (C.B.) \\ * Correspondence: Laura.Bechtold@hhu.de; Tel.: +49-0211-811-1597
}

Received: 16 May 2018; Accepted: 21 June 2018; Published: 1 July 2018

\begin{abstract}
The concreteness effect (CE) describes a processing advantage for concrete over abstract words. Electrophysiologically, the CE manifests in higher N400 and N700 amplitudes for concrete words. The contribution of the stimulus-inherent imageability to the electrophysiological correlates of the CE is not yet fully unraveled. This EEG study focused on the role of imageability irrespective of concreteness by examining the effects of training-induced visual imageability on the processing of novel words. In two training sessions, 21 healthy participants learned to associate novel words with pictures of novel objects as well as electron-microscopical structures and were additionally familiarized with novel words without any picture association. During a post-training EEG session, participants categorized trained novel words with or without picture association, together with real concrete and abstract words. Novel words associated with novel object pictures during the training elicited a higher N700 than familiarized novel words without picture-association. Crucially, this training-induced N700 effect resembled the CE found for real words. However, a CE on the N400 was found for real words, but no effect of imageability in novel words. The results suggest that the N400 CE for real words depends on the integration of multiple semantic features, while mere visual imageability might contribute to the CE in the N700 time window.
\end{abstract}

Keywords: N400; N700; concreteness; imageability; novel words; learning

\section{Introduction}

Language processing requires an association of a word's form with its referent's conceptual representation in semantic memory. Conceptual representations combine information taken from learning experience with the word and/or its referent and provide this information in the course of conceptual processing [1,2]. Depending on experiential differences concerning the words' referents, words are often classified as either concrete or abstract (for a review see [3]). Concrete words' referents (e.g., hammer) are perceivable with the external bodily senses. Abstract words refer to states or entities (e.g., harmony), which are not directly perceivable via external bodily senses, but rather arise from lexical information [4] or internal bodily senses (e.g., mental or emotional experience) [3,5-7].

The concreteness effect (CE) describes a processing advantage for concrete over abstract words in memory, comprehension and production tasks (for reviews see $[3,8,9]$ ). The dual-coding theory [4] explains the $\mathrm{CE}$ in terms of richer conceptual representations of concrete words, based on sensory as well as lexical information, while representations of abstract words rely on lexical information only. The context availability model [8] attributes the CE to an easier retrieval of a greater amount of conceptual information for concrete than abstract words. By now, novel approaches integrate dual-coding and context availability (e.g., the extended dual-coding theory) $[10,11]$, as they seem to 
highlight the two distinct but compatible semantic processes of concept representation and retrieval, which rely on different interacting neural correlates [3,12].

One component considered crucially relevant for processing differences between concrete and abstract words is their imageability. Dual-coding as well as context availability accounts assign concrete words a higher imageability in terms of conceptually integrated visual sensory information [9] and accessibility of mental images [13], respectively. Language processing advantages driven by imageability have been shown in children, who initially acquire [14-16] and subsequently learn to read [17] highly imageable words earlier and better. Moreover, concrete and/or highly imaginable word processing is often less severely impaired by clinical disorders like semantic dementia [18,19], dyslexia [20-25] and Alzheimer's disease [26]. Kellogg, et al. [27] showed that a concurrent visual working memory task impaired the performance of healthy young adults in a definition production task for concrete but not abstract words, further supporting the role of imageability in the CE. Therefore, it is not surprising that concreteness and imageability ratings are highly correlated [28] and the two terms are often used interchangeably [3,29].

In electroencephalography (EEG) studies, the CE becomes manifest in higher amplitudes of the N400 and N700 event-related potential (ERP) components for concrete in comparison to abstract words [30]. The N400 has been interpreted to reflect the strength of the activation of the semantic network and integration of semantic information (for extensive reviews see [31,32]). The frontally pronounced N700 has been linked to mental imagery processes [10,30,33-35]. Findings of Barber, et al. [36] question the role of imageability for the electrophysiological CE. They matched concrete and abstract words for imageability and found a reversed behavioral CE but still higher N400 amplitudes for concrete than abstract words. Therefore, the effects of imageability and concreteness on word processing seem to be at least partially dissociable.

One recent line of research made important contributions for disentangling the effects of imageability and concreteness on N400 and N700 amplitudes. Concrete and abstract words (as stand-alone stimuli, see [35]; or in a sentential context, see [34,37]) were processed in an image generation as well as in a lexical and/or surface-level processing task in order to manipulate stimulus- and task-driven imagery processes, respectively. Altogether, these studies suggest that word concreteness and imageability are distinct semantic features, which are integrated in the processing stage reflected by the N400. At the later processing stage reflected by the N700, mental images of words might be generated, but only when the task as well as the stimuli afford it (for a detailed discussion and information on methodological differences see [35]). Gullick, Mitra and Coch [35] interpret their findings based on the extended dual-coding theory $[10,11]$, and suggest that concreteness is not merely relying on sensorimotor information but includes lexically mediated information as well, while imageability is derived from (in their case visual) sensory information alone. In order to test this assumption, one could investigate the contribution of word imageability and concreteness to N400 and N700 modulations separately by employing stimuli with just one or the other semantic feature.

This study aimed at investigating the extent to which visual imageability untainted by concreteness modulates the N400 and N700 by using formerly meaningless, novel words that were either associated with visual stimuli during a training phase or not. In particular, in a two-day training, subjects learned to associate novel words with pictures of novel, unknown objects (OPic; see [38]) or of electron-microscopical structures (SPic). The two types of pictorial stimuli were chosen because of the different types of visual information they provide and were thus expected to lead to differences in imageability between the associated word stimuli. More specifically, the OPic were expected to lead to higher imageability than the SPic as we chose them to more distinctively depict one coherent entity. As a control condition, participants learned novel words that were not associated with any visual stimulus (NoPic). We thereby manipulated the novel words' imageability, without introducing any additional (lexical or sensory) information possibly contributing to the CE [3,39-41]. In a post-training EEG session, we examined the processing of the novel words while participants performed a concreteness-judgment task. 
The results for the N400 could help to elucidate the role of imageability for the CE. If imageability itself contributes to the N400 CE, training-induced higher visual imageability should lead to higher N400 amplitudes. If, however, the N400 CE depends on an integrative interaction of sensorimotor and lexically coded features underlying word concreteness [32,35,42], no effect should be seen at this processing stage. For the N700, we expected to see larger amplitudes for higher imageability, as the task used in our study was designed to afford imagery processes [35]. Finally, as the effect of visual imageability on word processing presumably contributes to the electrophysiological $\mathrm{CE}$, we presented concrete and abstract real words intermixed with the novel words in our study. The aim was to elicit a classical CE in the N400 and N700 time windows within the same experimental paradigm and qualitatively compare the $\mathrm{CE}$ in real words with the effects of imageability on novel words.

\section{Materials and Method}

\subsection{Participants}

Twenty-four healthy German native speakers (age from 19 to 34 years) took part in the study. Three participants were excluded from the analyses due to a poor learning performance and thus too few trials for the EEG analyses ( $<20$ for at least one experimental condition). The remaining 21 participants ( 10 women; mean age $=24.8$ years, $S D=4.1$ years) had normal or corrected to normal vision and were right handed as indicated by the Edinburgh Handedness Inventory [43] (scores between 0.55 and $1, M=0.88, S D=0.14$ ). All participants gave their written informed consent. After participation, they received course credit or monetary compensation. This study was in line with the declaration of Helsinki and was approved by the ethics committee of the Faculty of Mathematics and Natural Sciences.

\subsection{Material}

\subsubsection{Visual Stimuli}

The visual stimuli were 8-bit color JPG images of 15 unfamiliar objects (OPics) and 15 electronmicroscopical pictures of structures (SPics). The objects were built of a construction toy $\left(\mathrm{K}^{\prime} \mathrm{NEX} \mathrm{X}^{\mathrm{TM}}\right)$ and had already been used in previous training studies [38,44-47]. For each object, photographs from four isometric perspectives were available. The electron-microscopical pictures were acquired via google image search and consisted of different living and non-living structures (e.g., legionella, rocks, asbestos, skin). They were each cut into four partially overlapping segments and a slight vignette, extracted from the object picture backgrounds, was added. Electron-microscopical images were originally monochrome. The color information of the OPics was extracted, smoothed and transferred onto the SPics via the GNU Image Manipulation Program (GIMP, version 2). The mean brightness (measured with the pictures' histograms, from $0=$ white to $255=$ black with GIMP) of the 60 OPics $(M=176.77$, $S D=8.49)$ and 60 SPics $(M=176.77, S D=8.46)$ was carefully matched, $t(118)=0.001, p=0.999$.

\subsubsection{Verbal Stimuli}

The 60 word-like pseudo-words used as novel words in this study were created by changing two to three letters in real German words, following phonological rules (e.g., Himmar, Neribon). This pool of stimuli was divided into four subsets, each including 15 words. Each subset was assigned to one of the experimental conditions, namely OPic, SPic, NoPic (familiarized in the training but without associated pictures, served as a lexical baseline condition) and New (only used as filler stimuli for the EEG task, see below). The novel words in these four subsets were matched for the number of letters $\left(M_{\text {OPic }}=7.67, S D_{\text {OPic }}=0.90 ; M_{\text {SPic }}=7.67, S D_{\text {SPic }}=0.90 ; M_{\text {NoPic }}=7.73\right.$, $S D_{\mathrm{NoPic}}=0.88 ; M_{\mathrm{New}}=7.87, S D_{\mathrm{New}}=0.92 ;$ Kruskal-Wallis-test for independent samples, $H(3)=0.250$, $p=0.969)$ and syllables $\left(M_{\mathrm{OPic}}=2.53, S D_{\mathrm{OPic}}=0.52 ; M_{\mathrm{SPic}}=2.53, S D_{\mathrm{SPic}}=0.52 ; M_{\mathrm{NoPic}}=2.53\right.$, $S D_{\mathrm{NoPic}}=0.52 ; M_{\mathrm{New}}=2.47, S D_{\mathrm{New}}=0.52 ;$ Kruskal-Wallis-test for independent samples, $H(3)=0.197$, 
$p=0.978)$. The 60 real words additionally used in the EEG concreteness-judgment task consisted of 30 concrete and 30 abstract words. They were also matched for the number of letters $\left(M_{\text {concrete }}=7.07\right.$, $S D_{\text {concrete }}=1.02 ; M_{\text {abstract }}=7.10, S D_{\text {abstract }}=1.79 ;$ Kruskal-Wallis-test for independent samples, $H(1)=0.052, p=0.820)$ and syllables $\left(M_{\text {concrete }}=2.40, S D_{\text {concrete }}=0.50 ; M_{\text {abstract }}=2.47, S D_{\text {abstract }}=0.51\right.$; Kruskal-Wallis-test for independent samples, $H(1)=0.267, p=0.605)$. Concrete and abstract real words were additionally matched for their lexical frequency as assessed via a word database of the university of Leipzig, (http: / / wortschatz.uni-leipzig.de, 20 March 2015; $M_{\text {concrete }}=1726.68, S D_{\text {concrete }}=1698.92$; $M_{\text {abstract }}=1746.57, S D_{\text {abstract }}=2124.28 ;$ Kruskal-Wallis-test for independent samples, $H(1)=0.514$, $p=0.473$ ). All real words were rated regarding eight different psycholinguistic variables (Concreteness, Imageability, Arousal, Valence and their association with Action, Emotion, Perception and Thinking) by a sample of 39 ( 28 female) participants aged between 18 years and 44 years $(M=25.31$ years, $S D=6.73$ ) in a preceding rating-study (see Table S1 in the Supplementary Materials for descriptive and inferential statistics).

\subsection{Procedure}

\subsubsection{Training Sessions}

The training sessions took place with one or two participants in one room of the Department of Biological Psychology at Heinrich Heine University Düsseldorf. The training period for each participant included two training sessions on separate days with two training-blocks in each session, run with PsychoPy (version 1.81.03, avaliable online: http:/ /www.psychopy.org/changelog.html\# psychopy-1-81-03) [48] on a Fujitsu Lifebook A512. A two-minute break separated the two blocks. In both blocks, all OPic, SPic and NoPic words were presented four times each for $5000 \mathrm{~ms}$ in a randomized order. The ISI was set to $500 \mathrm{~ms}$. Within each block, each OPic word was combined once with each of the four pictures of the assigned object taken from different perspectives. Similarly, each SPic word was combined once with each of the four sections of one structure picture. In this way, each OPic and each SPic word appeared four times per block and thus eight times per training and each OPic word could be associated with one object and each SPic word could be associated with one structure. NoPic words were presented as often as the OPic and SPic words, but they appeared alone on the computer screen without any additional picture. Participants were asked to memorize the presented words and, for the OPic and SPic words, their associated pictures. Each block took about $15 \mathrm{~min}$ to complete. Participants were told that learning performance checks would be conducted after the training session. At first, free reproduction was assessed followed by a multiple-choice and picture assignment questionnaire (for details see Section 2.3.3).

\subsubsection{EEG Session}

EEG was acquired individually in an electrically shielded EEG chamber in the department of Biological Psychology at Heinrich Heine University Düsseldorf. During the EEG session, concrete and abstract real words as well as the novel words were presented intermixed in three blocks. The novel words included the 45 words that appeared during the training, as well as the 15 non-trained novel words (New condition). In each block, each novel and real word was presented once, and the order of presentation was randomized.

The participants' task was to judge whether the real and novel words were either concrete or abstract. This task was chosen because the definitions of concrete and abstract could be applied to both the real and novel words. This made it possible to use the same task for both types of words, which was especially important, as the words appeared intermixed. In the instructions for the participants, concrete was defined as referring to something perceivable via the senses (e.g., sight, touch) including real concrete words as well as the newly learned OPic and SPic words, which referred to the associated picture stimuli. Abstract was defined as referring to something not perceivable via the senses, including 
real abstract words as well as the NoPic and New words. The latter were introduced as filler stimuli to provide the same number of real and novel words.

Each trial started with a fixation cross with a jittered duration between $1200 \mathrm{~ms}$ and $1600 \mathrm{~ms}$. Then the word was presented for $800 \mathrm{~ms}$, followed by a jittered blank screen of $300 \mathrm{~ms}$ to $500 \mathrm{~ms}$ duration. Afterwards, the assignment of the left and right Ctrl-button of a computer keyboard to the concrete and abstract response option appeared on the screen. The button-response assignment varied randomly between trials to make sure that motor preparation would not confound the recorded ERPs. The inter-trial interval was again randomly jittered between $300 \mathrm{~ms}$ and $500 \mathrm{~ms}$. Participants had the possibility to take a self-paced break every 20 trials. Participants were asked to keep their left and right index fingers on the Ctrl-buttons in order to reduce movement artifacts. The EEG task took about $30 \mathrm{~min}$ to complete. Subsequent to the EEG task, participants completed the multiple-choice and picture assignment learning performance checks.

\subsubsection{Learning Performance Questionnaires}

Different questionnaires assessed the participants' learning performance. A free reproduction task tested the ability to recall the learned words freely after both training sessions. Following each training and the EEG session, a multiple-choice questionnaire with a list of all words tested the participants' ability to assign the novel words to their category (based on the training condition associated with object, structure and no picture). In an attached picture assignment task, participants were additionally asked to assign each novel word to the printed photographs of the objects/structures. In the learning performance tests, participants could reach one point per correct free reproduction and assignment of the novel words to their category (OPic, SPic, NoPic) or picture (OPic, SPic), respectively. In all versions of the multiple-choice questionnaire, the order of the words was randomized. For each category and learning performance measure, the percentage of correct reproduction and assignments was calculated.

\subsection{EEG Recording and Preprocessing}

\subsubsection{Recording}

EEG was recorded via 28 silver/silver chloride ring-electrodes, on a textile cap with pre-mounted holders (actiCap; Brainproducts $\mathrm{GmbH}$, Germany) following the extended 10-20 system [49] (electrode sites were F7, F3, Fz, F4, F8, FC5, FC1, FC2, FC6, T7, C3, Cz, C4, T8, CP5, CP1, CP2, CP6, P7, P3, $\mathrm{Pz}, \mathrm{P} 4, \mathrm{P} 8, \mathrm{PO} 9, \mathrm{O} 1, \mathrm{Oz}, \mathrm{O} 2$, and PO10). Additionally, two electrodes at the outer canthi of the eyes and one above and below the right eye, respectively, recorded horizontal and vertical eye movements. The ground electrode was attached at electrode site AFz and the online reference was attached to the nose. Impedances were kept below $5 \mathrm{k} \Omega$. EEG data were amplified via a BrainAmp DC amplifier (BrainProducts GmbH, Gilching, Germany). The Brain Vision Recorder software (Version 1.20.0506, Brain Products $\mathrm{GmbH}$ ) was used for data acquisition with a sampling rate of $1000 \mathrm{~Hz}$ and an online lowpass filter of $100 \mathrm{~Hz}$ on a Windows 10 Dell Intel Premium PC. The software Presentation (Version 17.0, Neurobehavioral Systems Inc., Albany, CA, USA) on a Windows 10 Dell Intel Premium PC controlled the timing of stimulus presentation during the EEG session on a $22^{\prime \prime}$ LED Dell monitor with $1680 \times 1050$-pixel resolution and a refresh rate of $60 \mathrm{~Hz}$. The software also recorded the participants' responses given via a Microsoft USB keyboard.

\subsubsection{Preprocessing}

EOG electrodes were re-referenced bipolarly and scalp electrodes were referenced to an average reference including all electrodes $(\mathrm{C} 3, \mathrm{C} 4, \mathrm{CP} 3, \mathrm{CP} 4, \mathrm{CPz}, \mathrm{Cz}, \mathrm{F} 3, \mathrm{~F} 4, \mathrm{~F} 7, \mathrm{~F} 8, \mathrm{FC} 3, \mathrm{FC} 4, \mathrm{FC}$, FT7, FT8, Fz, P3, P4, P7, P8, PO3, PO4, PO7, PO8, POz, Pz), except for T7 and T8, which showed extensive muscle artifacts in some participants. Next, data underwent a global direct current detrend [50]. We applied butterworth zero-phase filters with a highpass threshold of $0.5 \mathrm{~Hz}$ and a lowpass threshold 
of $20 \mathrm{~Hz}$, both with $24 \mathrm{~dB} /$ Oct. Additionally, a $50 \mathrm{~Hz}$ notch-filter was applied. After a classic ICA in semiautomatic mode on $120 \mathrm{~s}$ of the data, components including sharp, frontally pronounced positive deflections caused by blinking were detected by visual inspection and removed from the signal via an ICA back transformation. For 18 participants, one single component could be identified depicting the eye blink artifact. For the remaining three participants two or three components were excluded before the back transformation. Continuous data were segmented starting $300 \mathrm{~ms}$ pre- and ending $1200 \mathrm{~ms}$ post-stimulus onset. After a baseline correction for the $300 \mathrm{~ms}$ pre-stimulus interval, an automatic artifact rejection was applied with the following parameters: a maximal allowed voltage step of $50 \mu \mathrm{V} / \mathrm{ms}$, a maximal/minimal amplitude difference between the highest and the lowest data point of $100 / 0.1 \mu \mathrm{V}$ in $100 \mathrm{~ms}$, and a maximally/minimally allowed amplitude of $\pm 100 \mu \mathrm{V}$. Subsequently, artifact-free segments were divided into the experimental conditions OPic, SPic, NoPic and New for novel words, and concrete and abstract for real words. In the OPic, SPic and NoPic conditions, only those novel words were included, which participants correctly assigned to their training condition in the multiple-choice questionnaire after the EEG session. This resulted in a mean number of 38.8 trials $(S D=7.4)$ in the OPic, 37.6 trials $(S D=7.2)$ in the SPic and 36.5 trials $(S D=8.4)$ in the NoPic condition entering into the averaged ERP waveforms. In real-word conditions, all artifact-free segments (concrete: $M=89.2, S D=2.1$; abstract: $M=89.0, S D=2.2$ ) entered into the average ERP waveforms.

\subsubsection{ERP Analyses}

Nine electrodes (F3, Fz, F4, C3, Cz, C4, P3, Pz, P4) equally distributed across the head were chosen for the ERP analyses. ERP time windows were set after visual inspection of the grand average ERP waveforms and in line with previous studies $[30,31]$. For the N400, the mean amplitude was extracted from the time window between $300 \mathrm{~ms}$ and $500 \mathrm{~ms}$. The N700 is more a slow wave rather than a clearly defined ERP component, and the visual inspection of our data suggested different result patterns early and late in the N700 time window between $500 \mathrm{~ms}$ and $900 \mathrm{~ms}$. We thus split the time window and analyzed an early N700 (from the $500 \mathrm{~ms}$ to $700 \mathrm{~ms}$, compare, e.g., [34,35,51]) and a late N700 (from $700 \mathrm{~ms}$ to $900 \mathrm{~ms}$ ) separately (compare, e.g., [36,52]). Novel words from the New condition were excluded from ERP analyses as they were only introduced as filler stimuli and we were not interested in studying old/new ERP effects, which are typically very pronounced $[53,54]$.

\subsection{Statistical Data Analyses}

Data analysis was conducted with IBM SPSS Statistics (version 23, IBM corporation, Armonk, NY, USA). Behavioral learning and concreteness-judgment performance as well as electrophysiological data were analyzed with different repeated measures analyses of variance (ANOVA). If the Mauchly test indicated a violation of the assumption of sphericity, Greenhouse-Geisser correction was applied and corrected degrees of freedom and $p$-values will be reported. For significance, an $\alpha$-level of 0.05 was assumed. Post-hoc pairwise comparisons were Bonferroni corrected.

\section{Results}

\subsection{Behavioral Data}

\subsubsection{Learning Performance}

Free Reproduction

In order to check how well participants learned the novel words, a Category (OPic, SPic, NoPic) $\times$ Session (first and second training session) repeated measures ANOVA was conducted on the performance in the free reproduction task (see Figure 1, left). This analysis revealed that the Category did not have a significant effect on the percentage of correct free reproductions, $p=0.211$. Correct free reproductions significantly increased from the first to the second training session, $F(1,20)=44.607$, $p<0.001, \eta_{\mathrm{p}}{ }^{2}=0.690$. The Category $\times$ Session interaction was significant, $F(1.450,28.992)=3.784$, 
$p=0.047, \eta_{\mathrm{p}}{ }^{2}=0.159$. Paired $t$-tests revealed that the performance increase was significant for all novel word categories, all $p<0.001$, with the largest increase for OPic, followed by SPic and NoPic.

\section{Multiple-Choice}

Performance in the multiple-choice test (see Figure 1, middle) served as a second measure of learning of the novel words. This measure was not only applied after each of the two learning sessions, but also after the EEG session. A Category (OPic, SPic, NoPic) $\times$ Session (first and second training session, EEG session) repeated measures ANOVA showed that neither the main effect of Category nor the Category $\times$ Session interaction were significant for the multiple-choice learning performance, both $p \geq 0.078$. We found a significant main effect of Session, $F(1.424,28.483)=15.923, p<0.001, \eta_{\mathrm{p}}{ }^{2}=0.443$. Pairwise comparisons showed a significant performance increase from the first to the second training session and from the first training to the EEG session, $p=0.002$ and $p<0.001$, respectively. The second training session and the EEG session did not differ significantly, $p=1.000$.

\section{Picture Assignment}

A Category (OPic, SPic) $\times$ Session (first and second training session, EEG session) repeated measures ANOVA was performed on the performance in the picture assignment test (see Figure 1, right), with the aim of determining how well participants learned to associate the novel words with the respective pictures. The ANOVA showed that neither the main effect of Category nor the Category $\times$ Session interaction affected the percentage of correct picture-assignments significantly, both $p \geq 0.511$. Again, the Session had a significant effect, $F(1.234,24.673)=17.753, p<0.001, \eta_{p}{ }^{2}=0.022$. Pairwise comparisons revealed a significant increase in correct assignments from the first to the second training session, and from the first training to the EEG session, $p=0.002$ and $p<0.001$, respectively. The second training and EEG session did not differ significantly, $p=1.000$.

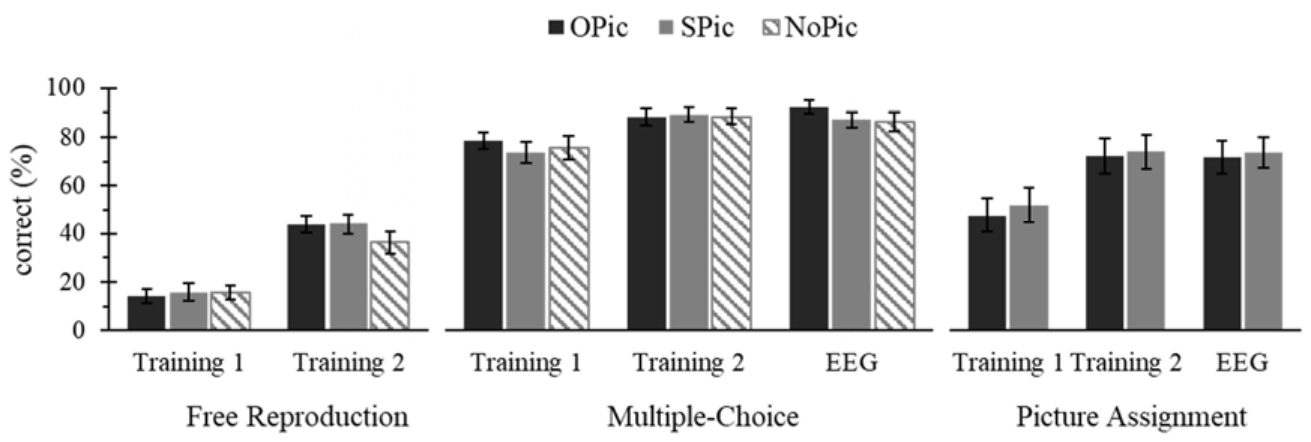

Figure 1. Novel word learning performance. The mean ( \pm one standard error for $n=21$ ) learning performance (\% correct) for the free reproduction task (left); multiple-choice questionnaire (middle) and picture assignment (right); separately for novel words associated with object (OPic), structure (SPic) or no picture (NoPic). Please note that the latter two learning measures were additionally acquired after the EEG session.

\subsubsection{Concreteness-Judgment Task in the EEG Session}

Error rates were calculated as the percentage of wrong responses of all given responses and were averaged separately for each experimental condition (real word Concreteness: concrete and abstract; novel word Category: OPic, SPic, NoPic).

Error Rates for Real Words

Mean error rates were $2.3 \%(S E=0.7 \%)$ for concrete words and $3.2 \%(S E=0.7 \%)$ for abstract words. A paired $t$-test did not reveal a significant difference between concrete and abstract words, $t(20)=-1.057, p=0.303$. 
Error Rates for Novel Words

Mean error rates were descriptively smallest in response to OPic $(M=8.3 \%, S E=2.2 \%)$, followed by SPic $(M=12.3 \%, S E=2.6 \%)$ and NoPic $(M=17.3 \%, S E=4.0 \%)$. In a repeated measures ANOVA the effect of the Category on error rates did not reach significance, $p=0.068$.

\subsection{Electrophysiological Data}

\subsubsection{ERP Effects for Real Words}

Firstly, we aimed at replicating the well-known CE for real words with our experimental paradigm and setup. Therefore, amplitudes of the N400 and the early and late N700 were analyzed via repeated measures ANOVAs with the factor Concreteness (concrete, abstract) and the topographical factors Frontality (frontal, central, parietal) and Laterality (left, middle, right). Figure 2 shows the grand averages for concrete and abstract words for all analyzed electrodes. Inferential statistics are listed in Table 1. Descriptive statistics $(M, S E)$ for the amplitudes of the ERP components elicited by concrete and abstract words at each electrode site can be found in Table S2 in the Supplementary Materials. Only main and interaction effects involving the factor Concreteness will be reported in the text.
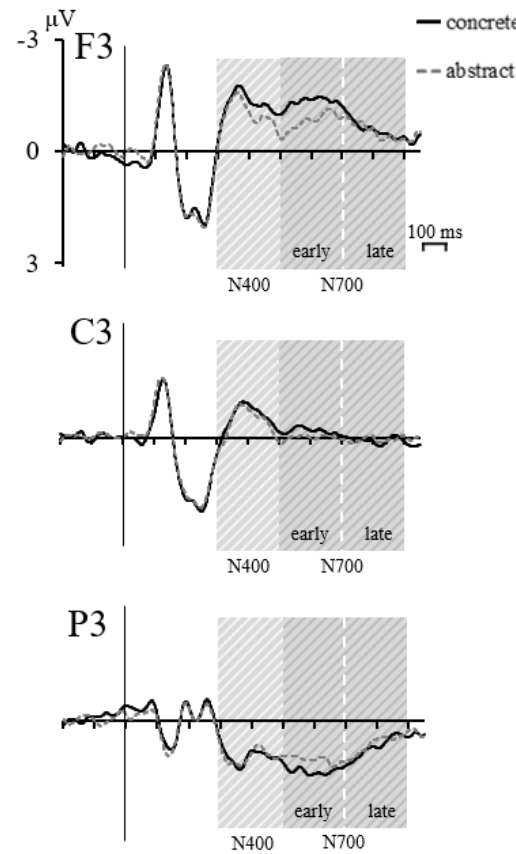
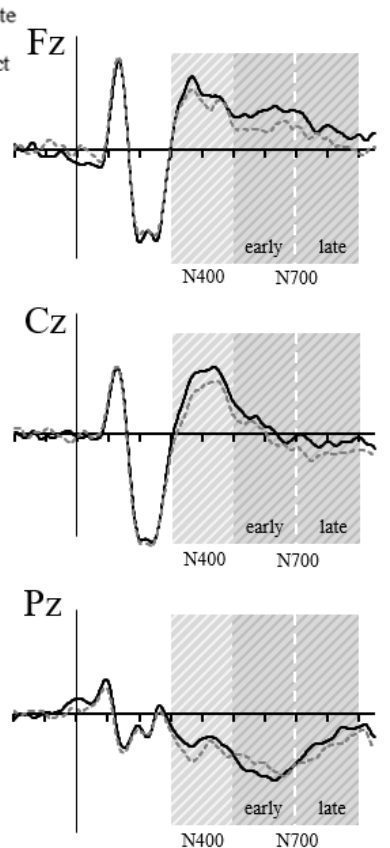
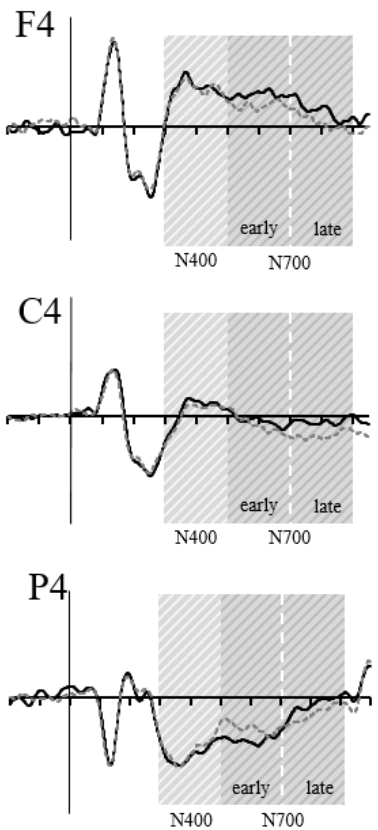

Figure 2. Real word ERPs. Grand averages $(n=21)$ of the ERP waveforms elicited by concrete and abstract words for all analyzed electrodes. The shaded areas mark the time windows of the N400 (300-500 ms), the early N700 (500-700 ms) and late N700 (700-900 ms).

\section{Real Word N400}

Concreteness had a significant effect on N400 amplitudes, $p=0.006$, with higher (i.e., more negative) amplitudes for concrete $(M=-0.259 \mu \mathrm{V}, S E=0.135 \mu \mathrm{V})$ than abstract words $(M=-0.124 \mu \mathrm{V}$, $S E=0.128 \mu \mathrm{V})$. The Concreteness $\times$ Laterality interaction was significant, $p=0.023$. Subsequent paired $t$-tests comparing abstract and concrete words for each of the three levels of Laterality revealed a significantly higher (i.e., more negative) N400 amplitude for concrete words only at electrodes of the midline (mean difference: $-0.274 \mu \mathrm{V}, S E=0.062 \mu \mathrm{V}$ ), $t(20)=-4.442, p<0.001$. The differences were significant at neither the left side, nor at right side electrodes, both $p \geq 0.078$. Neither the two-way interaction Concreteness $\times$ Frontality nor the three-way interaction Concreteness $\times$ Frontality $\times$ Laterality reached significance, both $p \geq 0.060$. 


\section{Real Word N700}

Concreteness had a significant effect on early N700 amplitudes, $p=0.016$, with a higher (i.e., less positive) amplitude for concrete $(M=0.068 \mu \mathrm{V}, S E=0.093 \mu \mathrm{V})$ than abstract words $(M=0.171 \mu \mathrm{V}$, $S E=0.092 \mu \mathrm{V})$. The Concreteness $\times$ Frontality interaction was significant, $p=0.005$. Subsequent paired $t$-tests comparing abstract and concrete words for each of the three levels of Frontality revealed a significantly more negative early N700 amplitude for concrete words at frontal (mean difference: $-0.408 \mu \mathrm{V}, S E=0.117 \mu \mathrm{V}), t(20)=-3.500, p=0.002$, and central electrodes (mean difference: $-0.168 \mu \mathrm{V}$, $S E=0.066 \mu \mathrm{V}), t(20)=-2.549, p=0.019$. The pattern was inversed at parietal electrodes, where concrete words elicited a significantly more positive amplitude than abstract words (mean difference: $0.270 \mu \mathrm{V}, S E=0.119 \mu \mathrm{V}), t(20)=2.275, p=0.034$. Neither the two-way interaction Concreteness $\times$ Laterality nor the three-way interaction Concreteness $\times$ Frontality $\times$ Laterality reached significance, both $p \geq 0.363$.

Concreteness also had a significant effect on late N700 amplitudes, $p=0.002$, again with higher (i.e., less positive) amplitudes for concrete $(M=0.055 \mu \mathrm{V}, S E=0.064 \mu \mathrm{V})$ than abstract words $(M=0.252 \mu \mathrm{V}, S E=0.076 \mu \mathrm{V})$. The Concreteness $\times$ Laterality interaction was significant, $p=0.025$. Subsequent paired $t$-tests comparing abstract and concrete words for each of the three levels of Laterality revealed a significantly higher late N700 amplitude for concrete words at midline (mean difference: $-0.298 \mu \mathrm{V}, S E=0.088 \mu \mathrm{V}), t(20)=-3.387, p=0.003$, and right side (mean difference: $-0.300 \mu \mathrm{V}, S E=0.084 \mu \mathrm{V}), t(20)=-3.574, p=0.002$ electrodes. Amplitudes elicited by concrete and abstract words did not differ significantly at left side electrodes, $p=0.916$. Neither the two-way interaction Concreteness $\times$ Frontality nor the three-way interaction Concreteness $\times$ Frontality $\times$ Laterality reached significance, both $p \geq 0.656$.

Table 1. Real Word Analyses. Full inferential statistics of the $2 \times 3 \times 3$ repeated measures ANOVAs on N400, early N700 and late N700 amplitudes.

\begin{tabular}{lcccc}
\hline \multicolumn{1}{c}{ Effect } & $d f$ & $\boldsymbol{F}$ & $\boldsymbol{p}$ & $\boldsymbol{\eta}_{\mathbf{p}}{ }^{2}$ \\
\hline $\mathrm{N} 400(300-500 \mathrm{~ms})$ & & & & \\
\hline Concreteness & 1,20 & 9.566 & 0.006 & 0.324 \\
Concreteness $\times$ Frontality & $1.251,25.023$ & 0.472 & 0.540 & 0.023 \\
Concreteness $\times$ Laterality & $1.302,26.036$ & 5.203 & 0.023 & 0.206 \\
Concreteness $\times$ Frontality $\times$ Laterality & 4,80 & 2.363 & 0.060 & 0.106 \\
Frontality & $1.135,22.691$ & 21.042 & $<0.001$ & 0.513 \\
Laterality & 2,40 & 5.614 & 0.007 & 0.219 \\
Frontality $\times$ Laterality & $2.544,50.884$ & 2.136 & 0.116 & 0.096 \\
\hline early N700 $(500-700$ ms) & & & & \\
\hline Concreteness & 1,20 & 6.961 & 0.016 & 0.258 \\
Concreteness $\times$ Frontality & $1.173,23.451$ & 8.588 & 0.005 & 0.300 \\
Concreteness $\times$ Laterality & 2,40 & 1.039 & 0.363 & 0.049 \\
Concreteness $\times$ Frontality $\times$ Laterality & 4,80 & 1.019 & 0.403 & 0.048 \\
Frontality & $1.364,27.271$ & 37.281 & $<0.001$ & 0.651 \\
Laterality & 2,40 & 0.849 & 0.435 & 0.041 \\
Frontality $\times$ Laterality & $1.643,32.859$ & 3.326 & 0.057 & 0.143 \\
\hline late N700 $(700-900$ ms) & & & & \\
\hline Concreteness & 1,20 & 12.796 & 0.002 & 0.390 \\
Concreteness $\times$ Frontality & $1.142,22.845$ & 0.247 & 0.656 & 0.012 \\
Concreteness $\times$ Laterality & $1.346,26.920$ & 4.974 & 0.025 & 0.199 \\
Concreteness $\times$ Frontality $\times$ Laterality & 4,80 & 0.187 & 0.944 & 0.009 \\
Frontality & $1.314,26.286$ & 21.153 & $<0.001$ & 0.514 \\
Laterality & 2,40 & 1.456 & 0.245 & 0.068 \\
Frontality $\times$ Laterality & $2.178,43.553$ & 7.345 & 0.001 & 0.269 \\
\hline
\end{tabular}

\subsubsection{ERP Effects for Novel Words}

The main analysis examined the effects of the training-induced visual imageability of the novel words on linguistic processing. Repeated measures ANOVAs with the training-induced factor Category 
(OPic, SPic, NoPic) and the topographical factors Frontality (frontal, central, parietal) and Laterality (left, middle, right) were conducted on the amplitudes of the N400 and the early and late N700. Figure 3 shows the grand averages for OPic, SPic and NoPic words for all analyzed electrodes. Inferential statistics are listed in Table 2. Descriptive statistics $(M, S E)$ for the ERP components elicited by OPic, SPic and NoPic words at each electrode site can be found in Table S3 in the Supplementary Materials. Only main and interaction effects involving the factor Category will be reported in the text.
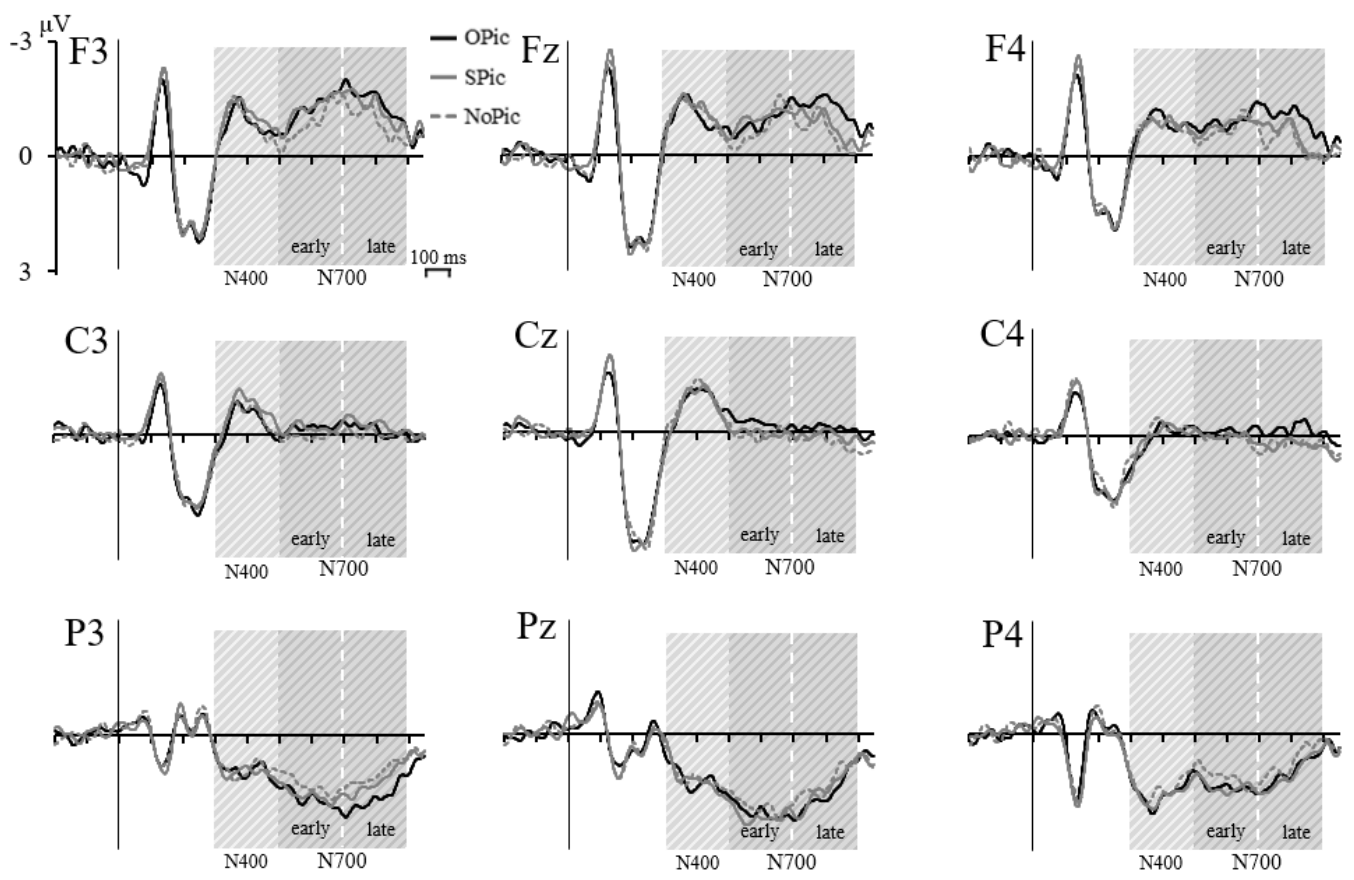

Figure 3. Novel word ERPs. Grand averages $(n=21)$ of the ERP waveforms elicited by novel words associated with object (OPic), structure (SPic) or no pictures (NoPic) for all analyzed electrodes. The shaded areas mark the time windows of the N400 (300-500 ms) and the early N700 (500-700 ms) and late N700 (700-900 ms).

\section{Novel Word N400}

For N400 amplitudes, neither the main effect of Category nor any of its interactions with the topographical factors reached significance, all $p>0.399$.

\section{Novel Word N700}

For the early N700, neither the main effect of Category nor any of its interactions with the topographical factors reached significance, all $p>0.141$.

For the late N700, the two-way interaction Category $\times$ Frontality was significant, $p=0.014$. In order to resolve this interaction, repeated measures ANOVAs investigated the effect of Category separately for each level of Frontality. The Category had a significant effect on the late N700 amplitudes at frontal $(p=0.008)$ and parietal $(p=0.043)$ but not at central $(p=0.066)$ electrode sites. Pairwise comparisons for the frontal electrodes showed that OPic words $(M=-1.244 \mu \mathrm{V}, S E=0.256 \mu \mathrm{V})$ elicited a significantly more negative amplitude than NoPic words $(M=-0.624 \mu \mathrm{V}, S E=0.227 \mu \mathrm{V}), p=0.021$. The comparisons of SPic $(M=-0.858 \mu \mathrm{V}, S E=0.277 \mu \mathrm{V})$ to OPic and NoPic did not reach significance, $p=0.087$ and $p=0.741$, respectively. The pattern was inverted at parietal electrodes, where OPic words $(M=1.374 \mu \mathrm{V}, S E=0.197 \mu \mathrm{V})$ elicited a more positive amplitude than NoPic words $(M=0.978 \mu \mathrm{V}$, $S E=0.172 \mu \mathrm{V})$ which was at trend level after Bonferroni correction, $p=0.099$. Again, the comparisons of SPic $(M=1.206 \mu \mathrm{V}, S E=0.214 \mu \mathrm{V})$ to OPic and NoPic were not significant, $p=0.396$ and $p=0.571$, respectively. The two-way interaction Category $\times$ Laterality was also significant, $p=0.047$. In order to 
resolve this interaction, repeated measures ANOVAs investigated the effect of Category separately for each level of Laterality. The Category had a significant effect on late N700 amplitudes only at right side electrodes, $p=0.027$ (left side and midline both $p \geq 0.133$ ). Pairwise comparisons for the right-side electrodes showed that OPic words $(M=-0.075 \mu \mathrm{V}, S E=0.130 \mu \mathrm{V})$ elicited a more negative amplitude than SPic words $(M=-0.235 \mu \mathrm{V}, S E=0.147 \mu \mathrm{V})$ and NoPic $(M=-0.178 \mu \mathrm{V}, S E=0.136 \mu \mathrm{V})$ which was at trend level after Bonferroni correction, $p=0.076$ and $p=0.080$, respectively, while the latter two did not differ significantly, $p=1.000$. Neither the Category main effect nor the three-way interaction Category $\times$ Frontality $\times$ Laterality were significant, both $p \geq 0.080$.

Table 2. Novel word analyses. Full inferential statistics of the $3 \times 3 \times 3$ repeated measures ANOVA on N400, early N700 and late N700 amplitudes as well as repeated measures ANOVAs with the factor Category (OPic, SPic, NoPic) resolving the significant interactions.

\begin{tabular}{|c|c|c|c|c|}
\hline Effect & $d f$ & $F$ & $p$ & $\eta_{p}^{2}$ \\
\hline \multicolumn{5}{|l|}{ N400 (300-500 ms) } \\
\hline Category & 2,40 & 0.559 & 0.576 & 0.027 \\
\hline Category $\times$ Frontality & $2.242,44.834$ & 0.135 & 0.895 & 0.007 \\
\hline Category $\times$ Laterality & 4,80 & 1.026 & 0.399 & 0.049 \\
\hline Category $\times$ Frontality $\times$ Laterality & 8,160 & 0.858 & 0.554 & 0.041 \\
\hline Frontality & $1.138,22.768$ & 21.611 & $<0.001$ & 0.519 \\
\hline Laterality & 2,40 & 2.387 & 0.105 & 0.107 \\
\hline Frontality $\times$ Laterality & $2.351,47.021$ & 1.924 & 0.151 & 0.088 \\
\hline \multicolumn{5}{|l|}{ early N700 (500-700 ms) } \\
\hline Category & 2,40 & 0.556 & 0.578 & 0.027 \\
\hline Category $\times$ Frontality & $2.115,42.309$ & 2.033 & 0.141 & 0.092 \\
\hline Category $\times$ Laterality & 4,80 & 0.415 & 0.797 & 0.020 \\
\hline Category $\times$ Frontality $\times$ Laterality & $5.126,102.523$ & 1.214 & 0.308 & 0.057 \\
\hline Frontality & $1.198,23.956$ & 28.031 & $<0.001$ & 0.584 \\
\hline Laterality & $1.328,26.560$ & 1.150 & 0.311 & 0.054 \\
\hline Frontality $\times$ Laterality & $1.747,34.947$ & 4.832 & 0.017 & 0.195 \\
\hline \multicolumn{5}{|l|}{ late N700 (700-900 ms) } \\
\hline Category & 2,40 & 2.698 & 0.080 & 0.119 \\
\hline Category $\times$ Frontality & $1.912,38.243$ & 4.917 & 0.014 & 0.197 \\
\hline \multicolumn{5}{|c|}{ Category $^{\text {a: }}$ Repeated measures ANOVA } \\
\hline frontal & 2,40 & 5.450 & 0.008 & 0.214 \\
\hline central & $1.567,31.341$ & 3.185 & 0.066 & 0.137 \\
\hline parietal & 2,40 & 3.408 & 0.043 & 0.146 \\
\hline Category $\times$ Laterality & 4,80 & 2.533 & 0.047 & 0.112 \\
\hline \multicolumn{5}{|c|}{ Category $^{\text {a }}$ : Repeated measures ANOVA } \\
\hline left side & 2,40 & 1.189 & 0.315 & 0.056 \\
\hline midline & $1.509,30.187$ & 2.255 & 0.133 & 0.101 \\
\hline right side & 2,40 & 3.941 & 0.027 & 0.165 \\
\hline Category $\times$ Frontality $\times$ Laterality & $4.367,87.338$ & 0.395 & 0.828 & 0.019 \\
\hline Frontality & $1.161,23.225$ & 27.382 & $<0.001$ & 0.578 \\
\hline Laterality & $1.500,30.009$ & 0.807 & 0.423 & 0.039 \\
\hline Frontality $\times$ Laterality & $2.164,43.278$ & 12.682 & $<0.001$ & 0.388 \\
\hline
\end{tabular}

\section{Discussion}

This study investigated the effect of visual imageability on linguistic processing untainted of lexical concreteness. In a two-day training paradigm, we induced visual imageability by letting participants associate novel words with two qualitatively different kinds of pictures. In a post-training EEG session, which also entailed real concrete and abstract words, we replicated the classical CE for real word processing, with higher (i.e., more negative) N400 and N700 amplitudes for concrete than abstract words. In the early N700 time window (500-700 ms), concrete words elicited significantly more negative amplitudes at frontal and central, but more positive amplitudes at parietal electrode sites. In the late N700 time window (700-900 ms), the CE was modulated by the laterality, with a significant $\mathrm{CE}$ at right side and midline, but no significant $\mathrm{CE}$ at left side electrodes. Concerning 
the processing of the novel words, we did not find effects of imageability on N400 amplitudes when comparing novel words associated with pictures and familiarized novel words without any picture association. For the late N700 time window, we found an imageability effect: Novel words associated with pictures of novel objects elicited significantly more negative amplitudes at frontal and more positive amplitudes at parietal electrode sites than non-imageable novel words. The direction of this effect at frontal electrode sites is in line with the hypothesis that a higher imageability contributes to the real word $\mathrm{CE}$ at this later conceptual processing stage reflected by the higher N700, while the N400 $\mathrm{CE}$ might reflect the interaction of sensorimotor and lexically coded conceptual features.

The higher N400 and N700 amplitudes for concrete in comparison to abstract real words are in line with the well-known CE and underline the suitability of our paradigm to uncover such semantic processing differences. The classical view explains N400 and N700 CEs on the basis of the extended dual-coding theory, namely to reflect a reliance on more easily accessible and qualitatively different information when processing concrete as compared to abstract concepts $[10,33]$. The concrete words used in the present study were rated higher in concreteness, imageability and their association with action and perception than abstract words (see Table S1 in the Supplementary Materials). The rating scores thus suggest that concrete conceptual representations are based on multi-modal information experienced with the external bodily senses, in line with previous psycholinguistic studies [3]. Hence, stronger semantic integration processes might explain the N400 CE, and stronger mental imagery processes driven by integrated sensory information the N700 CE [35] in our study. We did not find a behavioral CE on error rates, but a dissociation of behavioral and electrophysiological CEs is known from previous literature [36].

In order to interpret the electrophysiological results for the novel word processing against the background of their visual imageability, the validity of the training paradigm has to be examined. The participants' performance in the assessed learning questionnaires suggests that the training paradigm successfully established an association between the novel words and the assigned pictures. Free reproduction as well as multiple-choice and picture assignment performance showed an increase over the sessions for all three novel word categories. The novel words associated with pictures in our study seem to have additionally profited from their induced imageability, as indicated by the Category $\times$ Session interaction effect on the percentage of correct free reproductions. This is in line with another word-learning study, which traced back a learning advantage for concrete over abstract words to a stronger activation in the ventral anterior fusiform gyrus, which is involved in higher order visual processing [55].

The training-induced visual imageability did not affect the N400 amplitudes elicited during the processing of the novel words in the EEG session. As the novel words' imageability arose from mere visual information, they lacked concreteness in terms of lexically and multi-modally coded information, which seems to be crucial for the N400 CE [35,42]. An alternative explanation might be that the imageability induced for novel words was not sufficiently consolidated to elicit N400 effects. Other word-learning studies did neither find word-like N400 effects after a short training [56] nor before a consolidation period [57], while later ERP effects were found. However, our study consisted of two training sessions on separate days before the EEG acquisition and should thus have provided a sufficiently long period for consolidation. In addition, our analyses were restricted to those words for which the training condition was correctly recognized after the EEG assessment. Furthermore, Palmer, Macgregor and Havelka [29] found an N400 CE for words with merely lexically acquired concreteness (associated to written definitions) at the very same day. Hence, our data can reasonably be interpreted as being consistent with the hypothesis that the N400 CE relies on the interaction of several semantic features, to which isolated visual imageability does not contribute autonomously.

In line with our hypothesis, we found an effect of the training-induced imageability on late N700 amplitudes. This effect interacted with the topographical factors. OPic words elicited a significantly higher late N700 amplitude than NoPic words at frontal electrode sites. Amplitudes elicited by SPic processing were descriptively between those for OPic and NoPic but did not differ significantly from 
either of them. Only at electrode sites over the right hemisphere, OPic words elicited higher late N700 amplitudes than both SPic and NoPic at trend level. In a recent study on single-word processing, N700 imageability effects only arose when both, the task and the stimuli, afforded them [35]. The N700 result for the real words employed in the present study appears to indicate that the chosen task was appropriate for eliciting imagery processes. Concerning the lateralization of the N700 CE, previous studies yielded inconsistent findings, with more pronounced CEs either over the left [35] or right hemisphere ([34], but at occipital electrode sites), or no laterality difference at all [36,51,52]. However, in our study, the lateralization is in line with the stronger right hemispheric late N700 CE found for real abstract and concrete words.

Our pattern of results for novel words might suggest that the qualitative differences between the two kinds of employed pictures caused the late N700 modulation. The novel object pictures showed unique manmade objects, which formed a distinct entity: this characteristic might underlie advantages in early learning of imageable words [16]. The electron-microscopical pictures, in turn, although also containing coherent elements, were more heterogeneous and clearly less tangible. A possible alternative explanation for the graded late N700 effects might be a systematically weaker association of SPic words with their pictures. This explanation, however, seems implausible regarding the comparable performance in learning of SPic and OPic words. Notably, the deflection in the late N700 time window was positive at parietal electrodes, possibly reflecting a late positive component (LPC), usually interpreted to stand for the recollection of individual experience in linguistic processing [58,59]. In a word-learning study employing an old/new task, LPC amplitudes elicited by novel words were even higher after a consolidation period, while amplitudes decreased for familiar words [60]. The authors suggest that conscious recognition favors novel word learning. In our study, the LPC pattern was also found with more positive early N700 amplitudes elicited by concrete compared to abstract word processing.

As both the frontal N700 and the parietal LPC were delayed for novel (late N700) compared to real word CE (early N700), this might suggest a functional dissociation within the N700 time window. The relatively later N700 modulations by the imageability of novel words in our study might be due to their novelty, which could have led to a delayed processing in comparison to familiar concrete and abstract words. Previous findings concerning the temporal dynamics of the N700 for familiar words, however, are inconsistent, with findings of early onsets around $500 \mathrm{~ms}$ (compare e.g., [35,51]) as well as relatively late onsets only after $700 \mathrm{~ms}$ (compare e.g., [36]). Thus, exploring the functional role of different stages of the N700 might be a promising approach for further research.

Findings of Barber, et al. [36] challenge the classical interpretation of the N400 and N700 CE. In their ERP study, they controlled for concrete and abstract words' imageability and other psycholinguistic variables that are known to lead to concrete word processing advantages (i.e., familiarity, age of acquisition and context availability) and still found higher N400 and N700 amplitudes for concrete words. They suggest that the CE in these two ERP components is rather modulated by the degree of multimodality inherent to the underlying conceptual information. Following this suggestion, the lacking effect of the novel word category on N400 amplitudes might be explained by the unimodal visual information the words received during the training. At a later stage of semantic processing, additional information arising from mental imagery might have been processed, leading to effects of the novel word category in the late N700 time window. The tangible appearance of the novel object pictures might have led to an impression of an affordance inherent to graspable objects $[61,62]$ despite the lack of former experience with them. Linguistic processing might rely on this information [62,63], but rather at a later, more explicit processing stage, reflected by the N700.

In former studies, the electrophysiological CE could not easily be attributed to either the words' concreteness or imageability, as the two variables are highly correlated [3], and in most studies the terms were either used interchangeably or alone without controlling the other (for a counterexample see [36]). By modulating the visual imageability of former meaningless, novel words in a word learning paradigm, and thereby ruling out any possible confounds of word concreteness and other 
psycholinguistic variables, this study delivers insights into the isolated effect of words' imageability on linguistic processing. It seems that mere visual imageability plays a role at later explicit imagery processing stages (N700) but not in automatic semantic feature integration (N400). The effects in the N700 time window might also be explained by additional multi-modal information introduced by the novel object pictures and processed during mental imagery, which are not available at automatic stages of semantic processing.

Supplementary Materials: Supplementary materials can be found at http://www.mdpi.com/2227-9059/6/3/ 75/s1.

Author Contributions: Conceptualization, C.B., M.G. and L.B.; Methodology, C.B., M.G. and L.B.; Validation, C.B., M.G. and L.B.; Formal Analysis, L.B.; Investigation, L.B.; Resources, C.B.; Data Curation, L.B.; Writing-Original Draft Preparation, L.B.; Writing—Review \& Editing, C.B. and M.G.; Visualization, L.B.; Supervision, C.B. and M.G.; Project Administration, L.B.

Funding: This research received no external funding.

Acknowledgments: We thank Jenni Kuppe and Daniel Salz for their help in data acquisition and picture stimuli creation, Tatjana Maingardt for her help in the creation and rating of the verbal stimuli and Franziska Graham for her help in data acquisition.

Conflicts of Interest: The authors declare no conflict of interest.

\section{References}

1. Kiefer, M.; Pulvermuller, F. Conceptual representations in mind and brain: Theoretical developments, current evidence and future directions. Cortex 2012, 48, 805-825. [CrossRef] [PubMed]

2. Buccino, G.; Colage, I.; Gobbi, N.; Bonaccorso, G. Grounding meaning in experience: A broad perspective on embodied language. Neurosci. Biobehav. Rev. 2016, 69, 69-78. [CrossRef] [PubMed]

3. Hoffman, P. The meaning of 'life' and other abstract words: Insights from neuropsychology. J. Neuropsychol. 2016, 10, 317-343. [CrossRef] [PubMed]

4. Paivio, A. Mental Representations: A Dual-Coding Approach; Oxford University Press: Oxford, UK, 1986.

5. Ghio, M.; Vaghi, M.M.; Tettamanti, M. Fine-grained semantic categorization across the abstract and concrete domains. PLoS ONE 2013, 8, e67090. [CrossRef] [PubMed]

6. Binder, J.R.; Desai, R.H. The neurobiology of semantic memory. Trends Cogn. Sci. 2011, 15, 527-536. [CrossRef] [PubMed]

7. Troche, J.; Crutch, S.; Reilly, J. Clustering, hierarchical organization, and the topography of abstract and concrete nouns. Front. Psychol. 2014, 5, 360. [CrossRef] [PubMed]

8. Schwanenflugel, P.J.; Akin, C.; Luh, W.M. Context availability and the recall of abstract and concrete words. Mem. Cognit. 1992, 20, 96-104. [CrossRef] [PubMed]

9. Paivio, A. Dual coding theory: Retrospect and current status. Can. J. Psychol. Rev. Can. Psychol. 1991, 45, 255-287. [CrossRef]

10. Holcomb, P.J.; Kounios, J.; Anderson, J.E.; West, W.C. Dual-coding, context-availability, and concreteness effects in sentence comprehension: An electrophysiological investigation. J. Exp. Psychol. Learn. Mem. Cogn. 1999, 25, 721-742. [CrossRef] [PubMed]

11. Levy-Drori, S.; Henik, A. Concreteness and context availability in lexical decision tasks. Am. J. Psychol. 2006, 119, 45-65. [CrossRef] [PubMed]

12. Hoffman, P.; Binney, R.J.; Lambon Ralph, M.A. Differing contributions of inferior prefrontal and anterior temporal cortex to concrete and abstract conceptual knowledge. Cortex 2015, 63, 250-266. [CrossRef] [PubMed]

13. Schwanenflugel, P.J.; Stowe, R.W. Context availability and the processing of abstract and concrete words in sentences. Read. Res. Q. 1989, 24, 114-126. [CrossRef]

14. Rinaldi, P.; Barca, L.; Burani, C. A database for semantic, grammatical, and frequency properties of the first words acquired by italian children. Behav. Res. Methods Instrum. Comput. 2004, 36, 525-530. [CrossRef] [PubMed]

15. Bergelson, E.; Swingley, D. The acquisition of abstract words by young infants. Cognition 2013, 127, 391-397. [CrossRef] [PubMed] 
16. McDonough, C.; Song, L.; Hirsh-Pasek, K.; Golinkoff, R.M.; Lannon, R. An image is worth a thousand words: Why nouns tend to dominate verbs in early word learning. Dev. Sci. 2011, 14, 181-189. [CrossRef] [PubMed]

17. van der Veur, B.W. Imagery rating of 1000 frequently used words. J. Educ. Psychol. 1975, 67, 44-56. [CrossRef]

18. Jefferies, E.; Patterson, K.; Jones, R.W.; Lambon Ralph, M.A. Comprehension of concrete and abstract words in semantic dementia. Neuropsychology 2009, 23, 492-499. [CrossRef] [PubMed]

19. Hoffman, P.; Jones, R.W.; Lambon Ralph, M.A. Be concrete to be comprehended: Consistent imageability effects in semantic dementia for nouns, verbs, synonyms and associates. Cortex 2013, 49, 1206-1218. [CrossRef] [PubMed]

20. Crutch, S.J.; Warrington, E.K. Word form access dyslexia: Understanding the basis of visual reading errors. Q. J. Exp. Psychol. 2007, 60, 57-78. [CrossRef] [PubMed]

21. Barry, C.; Gerhand, S. Both concreteness and age-of-acquisition affect reading accuracy but only concreteness affects comprehension in a deep dyslexic patient. Brain Lang. 2003, 84, 84-104. [CrossRef]

22. Gerhand, S.; Barry, C. When does a deep dyslexic make a semantic error? The roles of age-of-acquisition, concreteness, and frequency. Brain Lang. 2000, 74, 26-47. [CrossRef] [PubMed]

23. Marchand, Y.; Friedman, R.B. Impaired oral reading in two atypical dyslexics: A comparison with a computational lexical-analogy model. Brain Lang. 2005, 93, 255-266. [CrossRef] [PubMed]

24. Jones, G.V. Predicability (ease of predication) as semantic substrate of imageability in reading and retrieval. Brain Lang. 2002, 82, 159-166. [CrossRef]

25. Jones, G.V. Deep dyslexia, imageability, and ease of predication. Brain Lang. 1985, 24, 1-19. [CrossRef]

26. Cuetos, F.; Arce, N.; Martinez, C.; Ellis, A.W. Word recognition in alzheimer's disease: Effects of semantic degeneration. J. Neuropsychol. 2017, 11, 26-39. [CrossRef] [PubMed]

27. Kellogg, R.T.; Olive, T.; Piolat, A. Verbal, visual, and spatial working memory in written language production. Acta Psychol. 2007, 124, 382-397. [CrossRef] [PubMed]

28. Paivio, A.; Yuille, J.C.; Madigan, S.A. Concreteness, imagery, and meaningfulness values for 925 nouns. J. Exp. Psychol. 1968, 76, 1-25. [CrossRef]

29. Palmer, S.D.; Macgregor, L.J.; Havelka, J. Concreteness effects in single-meaning, multi-meaning and newly acquired words. Brain Res. 2013, 1538, 135-150. [CrossRef] [PubMed]

30. Huang, H.W.; Federmeier, K.D. Imaginative language: What event-related potentials have revealed about the nature and source of concreteness effects. Lang. Linguist. (Taipei) 2015, 16, 503-515. [CrossRef] [PubMed]

31. Kutas, M.; Federmeier, K.D. Thirty years and counting: Finding meaning in the n 400 component of the event-related brain potential (erp). Annu. Rev. Psychol. 2011, 62, 621-647. [CrossRef] [PubMed]

32. Lau, E.F.; Phillips, C.; Poeppel, D. A cortical network for semantics: (de)constructing the n400. Nat. Rev. Neurosci. 2008, 9, 920-933. [CrossRef] [PubMed]

33. Kounios, J.; Holcomb, P.J. Concreteness effects in semantic processing: Erp evidence supporting dual-coding theory. J. Exp. Psychol. Learn. Mem. Cogn. 1994, 20, 804-823. [CrossRef] [PubMed]

34. West, W.C.; Holcomb, P.J. Imaginal, semantic, and surface-level processing of concrete and abstract words: An electrophysiological investigation. J. Cogn. Neurosci. 2000, 12, 1024-1037. [CrossRef] [PubMed]

35. Gullick, M.M.; Mitra, P.; Coch, D. Imagining the truth and the moon: An electrophysiological study of abstract and concrete word processing. Psychophysiology 2013, 50, 431-440. [CrossRef] [PubMed]

36. Barber, H.A.; Otten, L.J.; Kousta, S.T.; Vigliocco, G. Concreteness in word processing: Erp and behavioral effects in a lexical decision task. Brain Lang. 2013, 125, 47-53. [CrossRef] [PubMed]

37. Welcome, S.E.; Paivio, A.; McRae, K.; Joanisse, M.F. An electrophysiological study of task demands on concreteness effects: Evidence for dual coding theory. Exp. Brain Res. 2011, 212, 347-358. [CrossRef] [PubMed]

38. Bellebaum, C.; Tettamanti, M.; Marchetta, E.; Della Rosa, P.; Rizzo, G.; Daum, I.; Cappa, S.F. Neural representations of unfamiliar objects are modulated by sensorimotor experience. Cortex 2013, 49, 1110-1125. [CrossRef] [PubMed]

39. Altarriba, J.; Bauer, L.M.; Benvenuto, C. Concreteness, context availability, and imageability ratings and word associations for abstract, concrete, and emotion words. Behav. Res. Methods Instrum. Comput. 1999, 31, 578-602. [CrossRef] [PubMed]

40. Della Rosa, P.A.; Catricala, E.; Vigliocco, G.; Cappa, S.F. Beyond the abstract-concrete dichotomy: Mode of acquisition, concreteness, imageability, familiarity, age of acquisition, context availability, and abstractness norms for a set of 417 italian words. Behav. Res. Methods 2010, 42, 1042-1048. [CrossRef] [PubMed] 
41. Reilly, J.; Kean, J. Formal distinctiveness of high- and low-imageability nouns: Analyses and theoretical implications. Cogn. Sci. 2007, 31, 157-168. [CrossRef] [PubMed]

42. Huang, H.W.; Lee, C.L.; Federmeier, K.D. Imagine that! Erps provide evidence for distinct hemispheric contributions to the processing of concrete and abstract concepts. NeuroImage 2010, 49, 1116-1123. [CrossRef] [PubMed]

43. Oldfield, R.C. The assessment and analysis of handedness: The edinburgh inventory. Neuropsychologia 1971, 9, 97-113. [CrossRef]

44. Ruther, N.N.; Brown, E.C.; Klepp, A.; Bellebaum, C. Observed manipulation of novel tools leads to mu rhythm suppression over sensory-motor cortices. Behav. Brain Res. 2014, 261, 328-335. [CrossRef] [PubMed]

45. Ruther, N.N.; Tettamanti, M.; Cappa, S.F.; Bellebaum, C. Observed manipulation enhances left fronto-parietal activations in the processing of unfamiliar tools. PLoS ONE 2014, 9, e99401. [CrossRef] [PubMed]

46. Ghio, M.; Schulze, P.; Suchan, B.; Bellebaum, C. Neural representations of novel objects associated with olfactory experience. Behav. Brain Res. 2016, 308, 143-151. [CrossRef] [PubMed]

47. Bechtold, L.; Ghio, M.; Lange, J.; Bellebaum, C. Event-related desynchronization of mu and beta oscillations during the processing of novel tool names. Brain Lang. 2018, 177-178, 44-55. [CrossRef] [PubMed]

48. Peirce, J.W. Psychopy-Psychophysics software in python. J. Neurosci. Methods 2007, 162, 8-13. [CrossRef] [PubMed]

49. Chatrian, G.E.; Lettich, E.; Nelson, P.L. Ten percent electrode system for topographic studies of spontaneous and evoked eeg activities. Am. J. EEG Technol. 1985, 25, 83-92.

50. Hennighausen, E.; Heil, M.; Rosler, F. A correction method for dc drift artifacts. Electroencephalogr. Clin. Neurophysiol. 1993, 86, 199-204. [CrossRef]

51. Malhi, S.K.; Buchanan, L. A test of the symbol interdependency hypothesis with both concrete and abstract stimuli. PLoS ONE 2018, 13, e0192719. [CrossRef] [PubMed]

52. Nittono, H.; Suehiro, M.; Hori, T. Word imageability and N400 in an incidental memory paradigm. Int. J. Psychophysiol. 2002, 44, 219-229. [CrossRef]

53. Picton, T.W. The p300 wave of the human event-related potential. J. Clin. Neurophysiol. 1992, 9, 456-479. [CrossRef] [PubMed]

54. Marina, I.V.; Strelets, V.B. Verbal stimuli semantics and relevance of erps. Zhurnal Vyssh. Nerv. Deiat. Im. IP Pavlova 2010, 60, 22-31.

55. Mestres-Misse, A.; Munte, T.F.; Rodriguez-Fornells, A. Functional neuroanatomy of contextual acquisition of concrete and abstract words. J. Cogn. Neurosci. 2009, 21, 2154-2171. [CrossRef] [PubMed]

56. Lucas, H.D.; Hubbard, R.J.; Federmeier, K.D. Flexible conceptual combination: Electrophysiological correlates and consequences for associative memory. Psychophysiology 2017, 54, 833-847. [CrossRef] [PubMed]

57. Bakker, I.; Takashima, A.; van Hell, J.G.; Janzen, G.; McQueen, J.M. Tracking lexical consolidation with erps: Lexical and semantic-priming effects on $\mathrm{n} 400$ and lpc responses to newly-learned words. Neuropsychologia 2015, 79, 33-41. [CrossRef] [PubMed]

58. Van Petten, C.; Luka, B.J. Prediction during language comprehension: Benefits, costs, and erp components. Int. J. Psychophysiol. 2012, 83, 176-190. [CrossRef] [PubMed]

59. Strozak, P.; Bird, C.W.; Corby, K.; Frishkoff, G.; Curran, T. Fn400 and lpc memory effects for concrete and abstract words. Psychophysiology 2016, 53, 1669-1678. [CrossRef] [PubMed]

60. Palmer, S.D.; Havelka, J.; van Hooff, J.C. Changes in recognition memory over time: An erp investigation into vocabulary learning. PLoS ONE 2013, 8, e72870. [CrossRef] [PubMed]

61. Tucker, M.; Ellis, R. On the relations between seen objects and components of potential actions. J. Exp. Psychol. Hum. Percept. Perform. 1998, 24, 830-846. [CrossRef] [PubMed]

62. Borghi, A.M.; Riggio, L. Stable and variable affordances are both automatic and flexible. Front. Hum. Neurosci. 2015, 9, 351. [CrossRef] [PubMed]

63. Borghi, A.M.; Flumini, A.; Cimatti, F.; Marocco, D.; Scorolli, C. Manipulating objects and telling words: A study on concrete and abstract words acquisition. Front. Psychol. 2011, 2, 15. [CrossRef] [PubMed]

(C) 2018 by the authors. Licensee MDPI, Basel, Switzerland. This article is an open access article distributed under the terms and conditions of the Creative Commons Attribution (CC BY) license (http://creativecommons.org/licenses/by/4.0/). 